\title{
Fabrication and modeling of narrow capillaries for vacuum system gas inlets
}

\author{
Quaade, Ulrich; Jensen, Søren; Hansen, Ole
}

Published in:

Journal of Applied Physics

Link to article, DOI:

$10.1063 / 1.1829377$

Publication date:

2005

Document Version

Publisher's PDF, also known as Version of record

Link back to DTU Orbit

Citation (APA):

Quaade, U., Jensen, S., \& Hansen, O. (2005). Fabrication and modeling of narrow capillaries for vacuum system gas inlets. Journal of Applied Physics, 97(4), 044906. https://doi.org/10.1063/1.1829377

\section{General rights}

Copyright and moral rights for the publications made accessible in the public portal are retained by the authors and/or other copyright owners and it is a condition of accessing publications that users recognise and abide by the legal requirements associated with these rights.

- Users may download and print one copy of any publication from the public portal for the purpose of private study or research.

- You may not further distribute the material or use it for any profit-making activity or commercial gain

- You may freely distribute the URL identifying the publication in the public portal

If you believe that this document breaches copyright please contact us providing details, and we will remove access to the work immediately and investigate your claim 


\title{
Fabrication and modeling of narrow capillaries for vacuum system gas inlets
}

\author{
Ulrich J. Quaade \\ ICAT, Department of Physics, Technical University of Denmark, Building 312, 2800 Kgs. Lyngby, Denmark \\ Søren Jensen and Ole Hansen \\ MIC-Department of Micro- and Nanotechnology, Technical University of Denmark, Building 345E, 2800 \\ Kgs. Lyngby, Denmark
}

(Received 3 August 2004; accepted 14 October 2004; published online 21 January 2005)

\begin{abstract}
Micrometer-sized cylindrical capillaries with well-controlled dimensions are fabricated using deep reactive ion etching. The flow through the capillaries is experimentally characterized for varying pressures, temperatures, and diameters. For the parameters used, it is shown that the Knudsen number is in the intermediate flow regime, and Knudsen's expression for the flow fit the data well. The flow properties of the capillaries make them ideal for introducing gas into vacuum systems and in particular mass spectrometers. (C) 2005 American Institute of Physics. [DOI: 10.1063/1.1829377]
\end{abstract}

\section{INTRODUCTION}

Controlled introduction of gas into vacuum systems is important in studies and applications such as catalysis, molecular beams, and interaction of molecules with pure surfaces. For gas composition analysis, introduction of gas from a high-pressure region to a mass spectrometer working at a pressure below $10^{-7} \mathrm{~Pa}$ is necessary.

Kasemo ${ }^{1}$ produced quartz tube orifices with diameters of 5-7 $\mu \mathrm{m}$ and lengths of about $150 \mu \mathrm{m}$ by melting a quartz capillary tube in a hydrogen/oxygen flame. The quartz tubes are ideal for sampling gas to mass spectrometers with fast response time and minimum perturbation of the measured gas sample, and have been used for a variety of experiments. ${ }^{2-5}$ Despite the applicability, the quartz tube orifices suffer from limited reproducibility and knowledge about the exact geometry of the orifice, which prevents a detailed understanding and modeling of the flow. Especially for trusting the orifices as tools for gas analysis, for example, it is important to have a detailed understanding of the flow in the orifices and how it depends on for example gas composition and temperature.

In Refs. 6-8, long narrow channels are shown to have the possibility to affect the gas flow through gas-wall interactions. Both transient flow and separation effects are seen. The overall effect of gas-wall interactions is proportional to the number of wall-collisions suffered by a molecule traversing the channel, and is of the order $(l / d)^{2}$, where $l$ is the length and $d$ is the diameter. ${ }^{8}$ For use as gas probes, the gas-wall interactions should be minimized, and a small narrow capillary with a low value of $l / d$ is desirable.

In this article, deep reactive ion etching (DRIE) ${ }^{9}$ is used to produce narrow capillaries with well-controlled dimensions through silicon wafers. Each wafer can hold hundreds of capillaries, which can be separated after fabrication, and the method thus has obvious batch fabrication perspectives. The capillaries are perpendicular to the surface, and $l / d$ is between 5 and 25. The flow through the capillaries is experimentally characterized for varying diameters, temperatures, and pressures. The flow is modeled by Knudsen intermediate flow. Due to the simple geometry of the capillaries and the limited gas-surface interaction, the model describes the flow very well.

\section{EXPERIMENTS}

The capillaries are produced by employing the DRIE process in two steps, as illustrated in Fig. 1. First, the narrow capillary of diameter $2-5 \mu \mathrm{m}$ is etched from one side of the wafer to a depth larger than the final length. Second, the wafer is turned over and a large hole is etched from the other side until it meets the narrow capillary. When the etch rate is (a)

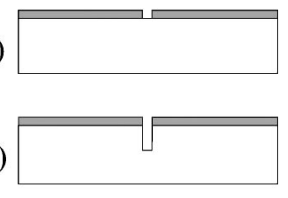

Photoresist $\square$ Silicon

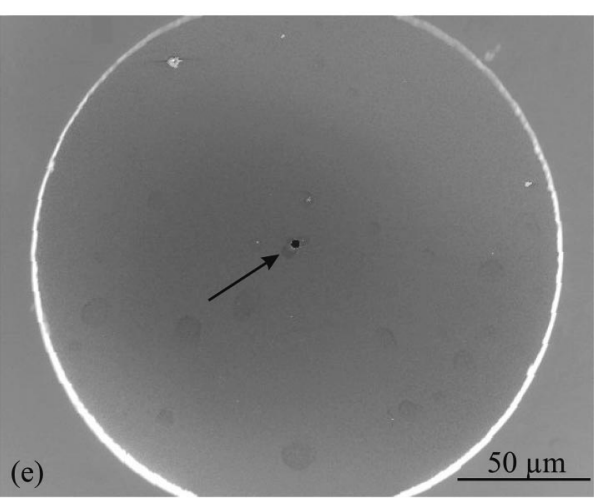

FIG. 1. Fabrication sequence for the capillaries: (a) photoresist mask is defined on one side of the wafer, (b) a narrow hole is etched, (c) the wafer is turned over and another etch mask containing a large hole is defined on the other side, (d) the large hole is etched until it meets the narrow hole, which then becomes the desired capillary, and (e) SEM image of the resulting silicon structure. The edge of the large hole is seen as the bright ring. The capillary, indicated by the arrow, is visible as a black dot in the bottom of the big hole. 

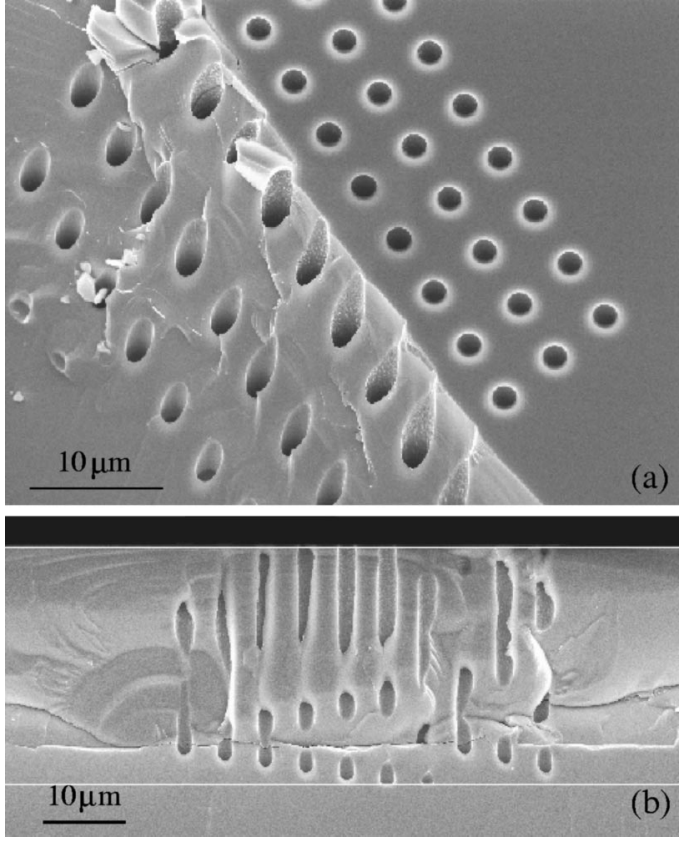

FIG. 2. Test structure showing an array of almost cylindrical holes etched into a silicon substrate: (a) cleaved across the holes and (b) cleaved parallel to the holes.

known, the etch can be continued until the narrow capillary has the desired length. The final structure is shown in Fig. 1(e).

The etch rate is not constant over the wafer but varies by about $\pm 5 \%$, giving a variation in the length of the capillaries of about $15 \mu \mathrm{m}$ if the wafer thickness is $300 \mu \mathrm{m}$. This is a significant uncertainty given that the produced capillaries usually have lengths between 10 and $75 \mu \mathrm{m}$. In an improved process using silicon-on-insulator substrates this variation can be completely eliminated.

In Fig. 2, a test structure of holes with diameter $d=2 \mu \mathrm{m}$ are etched to a depth of $30 \mu \mathrm{m}$. The holes are almost cylindrical and close inspection of different test structures gives an upper limit of the difference in diameter from top to bottom of about $0.5 \mu \mathrm{m}$. Scanning electron microscopy (SEM) investigations reveal that the larger capillaries have circular cross section, while the smaller capillaries may be slightly distorted. The SEM used gives accurate measurements of lateral dimensions within 5\%.

To characterize the flow through the capillaries, the chips are mounted on an aluminum interface block. On one side of the interface block, the chip containing the capillary is mounted by squeezing it onto a Viton o-ring. On the other side, the block is connected to a mass spectrometer. The mass spectrometer has a background pressure $p<10^{-10} \mathrm{~Pa}$ and is pumped with a turbo pump with pumping speed $S=56 \mathrm{l} / \mathrm{s}$. When the valve between the mass spectrometer and the tube is opened, the pressure rises until the amount of gas flowing through the capillary and the amount pumped by the turbo pump are the same. The amount of gas pumped by the turbo pump is proportional to the pressure $p$ and the pumping speed and is given by

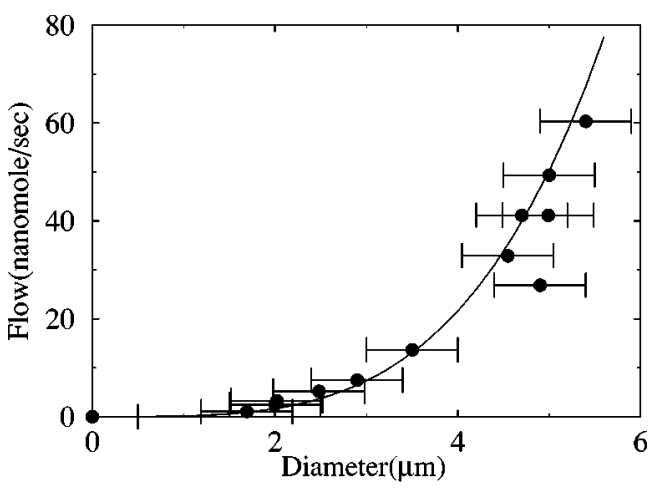

FIG. 3. Flow through the capillaries as function of diameter. The points show experimental flow data for different diameters of the capillary. The curve is a plot of Eq. (4) for $l=45 \mu \mathrm{m}$.

$$
\frac{d n}{d t}=\frac{p S}{R T}
$$

in terms of mole/second. $R$ is the gas constant and $T$ the temperature.

Since the pumping speed and the pressure gauge's sensitivity vary with gas composition, it is difficult to directly compare flow measurements for different gases. In the measurements presented here, the high-pressure side is atmospheric air at a pressure of $10^{5} \mathrm{~Pa}$. The pressure gauge used (cold cathode gauge) is calibrated for air. It is linear in the pressure range investigated in the present work and the statistical error is below 5\% on subsequent measurements. However, a systematic error might vary from $+60 \%$ to $-50 \%$ according to the manufacturors specifications. This means that we have to allow for a corresponding calibration factor for the flow obtained from Eq. (1).

The diameters of the capillaries are measured from SEM images and the molar flow is obtained from Eq. (1). Capillaries of different diameters give different molar flows. In Fig. 3, the flow through 13 different capillaries is displayed for diameters $1.6 \mu \mathrm{m}<d<5.2 \mu \mathrm{m}$. The flow increases strongly with increasing diameter.

To measure the dependence of flow on temperature, the chip containing the hole is mounted with a heater element on top. The heater element is a piece of low-resistance ( $<0.025 \Omega \mathrm{cm}$ ) silicon that is resistively heated. The temperature is measured with a thermocouple located between the heater element and the chip and the heating is controlled by a proportional integral differentiation algorithm implemented on a computer. The flow is again measured using Eq. (1). In Fig. 4, the dependence of the flow on temperature for a capillary with $d=3.1 \mu \mathrm{m}$ is shown. As the temperature is decreased from 430 to $325 \mathrm{~K}$ with a rate of $0.2 \mathrm{~K} / \mathrm{s}$, the molar flow increases by about $20 \%$. Similar behavior is observed for quartz tube orifices in Ref. 10.

Finally, the flow dependence on inlet pressure is investigated. For that purpose the capillary is mounted to allow flow from a fixed volume to a vacuum chamber. The pressure in the fixed volume is continuously measured using an absolute pressure gauge with accuracy better than $1 \%$. As gas flows through the capillary the pressure in the fixed volume decreases and the flow is obtained from 


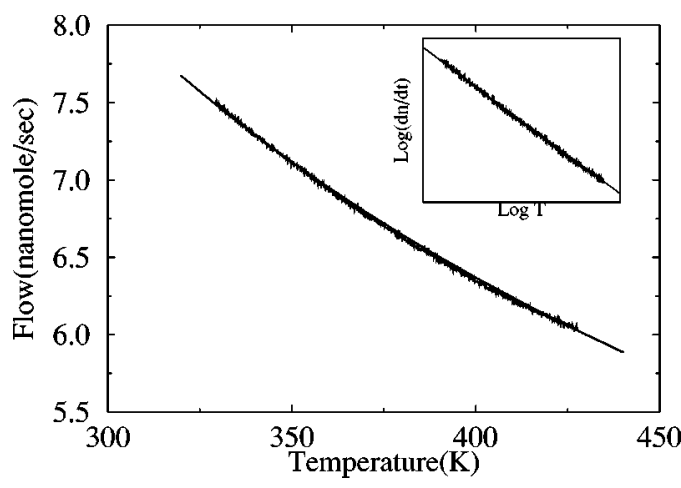

FIG. 4. Measured flow dependence on temperature: The fit curve is a plot of Eq. (4). The inset shows a log-log plot of the same data.

$$
\frac{d n}{d t}=\frac{V}{k T} \frac{d p}{d t},
$$

where $p$ is the pressure in the fixed volume, $V$ the volume, and $t$ is time. In this measurement there will be no systematic error on the flow. The result is displayed in Fig. 5 for a capillary with diameter $d=2.9 \mu \mathrm{m}$, as measured with SEM.

\section{DISCUSSION}

Gas flow in a tube of diameter $d$ can be characterized by the Knudsen number $\lambda / d$, where $\lambda$ is the mean free path of molecules in the gas. If $d / \lambda>110$, the flow is viscous, and if $d / \lambda<1$, the flow is molecular. In between, the flow is denoted intermediate. ${ }^{11}$ Expressions for the flow in a circular tube exist for all three regimes. For air at room temperature, the mean free path is given by

$$
\lambda=6.7 \times 10^{-3}[\mathrm{~Pa} \mathrm{~m}] / p,
$$

where $p$ is the pressure. ${ }^{11}$ This means that at atmospheric pressure the flow will be in the intermediate range for diameters $100 \mathrm{~nm}<d<7 \mu \mathrm{m}$. For realistic diameters of the capillary $(d>1 \mu \mathrm{m})$, the pressure at which the flow changes from intermediate to molecular is below $7 \times 10^{3} \mathrm{~Pa}$. In Fig. 6 , the capillary is shown with the two flow regimes. The inlet pressure is $p_{1}$, and at the pressure $p_{2}$, the flow changes from intermediate to molecular. The pressure in the vacuum chamber is denoted $p_{3}$.

Knudsen's expression for the flow in a circular tube in the intermediate flow regime is: ${ }^{11}$

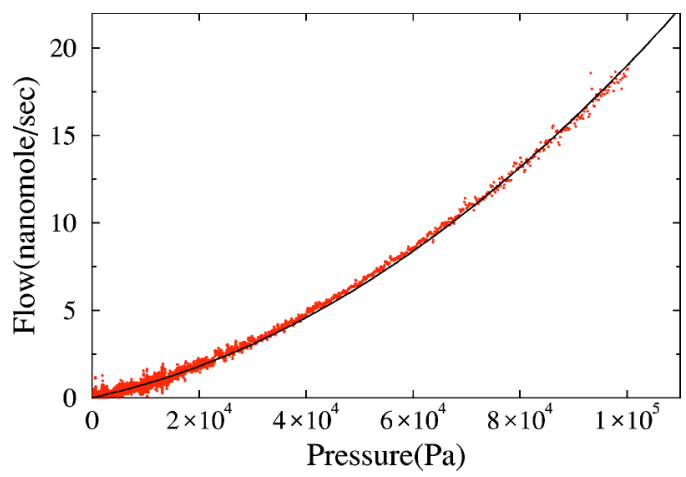

FIG. 5. Measured flow as function of the pressure. The fit curve is a plot of Eq. (4).

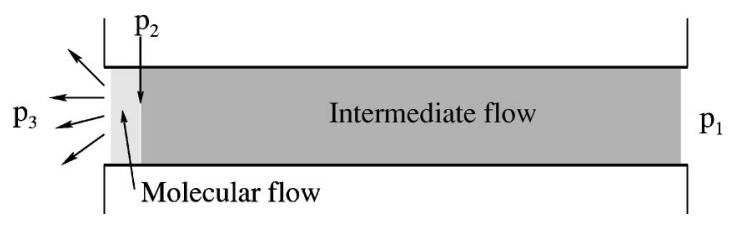

FIG. 6. Model of the capillary showing the high-pressure side and the vacuum side. The regions with intermediate flow and molecular flow are indicated. The inlet pressure is $p_{1}$ and $p_{3}$ is the pressure at the vacuum side. At the pressure $p_{2}$, the flow changes from intermediate to molecular.

$$
\frac{d n}{d t}=\frac{p_{1}-p_{2}}{R T} c
$$

with

$$
c=\frac{\pi}{128} \frac{\bar{p} d^{4}}{\eta l}+\frac{1}{3} \sqrt{\frac{\pi}{2}} \frac{d^{3}}{l v(T)} \frac{1+d v(T) \bar{p} / \eta}{1+1.24 d v(T) \bar{p} / \eta},
$$

where $\bar{p}=\left(p_{1}+p_{2}\right) / 2$ and $v(T)=\sqrt{M /(R T)}$. In the molecular flow regime

$$
\frac{d n}{d t}=\frac{p_{2}-p_{3}}{R T} \frac{1}{3} \sqrt{\frac{\pi}{2}} \frac{1}{\sqrt{v(T)}} \frac{d^{3}}{l} .
$$

Using Eq. (3), the expressions Eqs. (4) and (6) can be combined to obtain an analytical expression for the flow that includes both the intermediate and the molecular flow regime. Assuming $p_{1}=10^{5} \mathrm{~Pa}$ and $p_{3}=0$, it turns out that intermediate flow is dominating in more than about $95 \%$ of the length of the capillary for the dimensions in question. Thus, for the dimensions, temperatures, and pressures in this article, the molecular flow regime can be neglected and the flow is described by Eq. (4), setting $p_{2}=p_{3}$.

As noted earlier, there is an uncertainty in the measured value of the pressure in the mass spectrometer and the length of the capillaries. The flow predicted from Eq. (4) depends on the length as $l^{-1}$ and the measured flow depends linearly on the pressure $p$ in Eq. (1). Thus, when comparing the measured and predicted flows the combined uncertainty in the length $l$ and pressure $p$ reduces to an overall scaling factor between the measured flow from Eq. (1) and the flow predicted by Eq. (4). In the following fits the fitted values of $l$ are based on the (arbitrary) assumption that the measured pressure $p$ is correct. The obtained values for $l$ lie within the expected interval, but cannot be trusted as the true length of the investigated capillaries.

In Fig. 3, Eq. (4) is plotted as function of the diameter of the capillary for air with $M=29 \mathrm{~g} / \mathrm{mole}$ and $T=300 \mathrm{~K}$. The length $l=45 \mu \mathrm{m}$ is an average length of the capillaries and is used for the fitted curve. Within the uncertainties, the curve fits the data.

In Fig. 4, Eq. (4) is plotted as function of temperature for $l=50 \mu \mathrm{m}$. The diameter $d=3.16 \mu \mathrm{m}$ is chosen within the uncertainty to make the best fit to data.

Finally, in Fig. 5, Eq. (4) is plotted as function of the inlet pressure $p_{1}$ for $l=15.3 \mu \mathrm{m}$ and $d=2.9 \mu \mathrm{m}$. Again, these values are chosen within the uncertainty to make the best fit to the data. In all cases, good agreement between the predicted and the measured flows is obtained. 
In Ref. 10, it is found for quartz tube orifices that in the temperature range $300 \mathrm{~K}<T<600 \mathrm{~K}$, a log-log plot of the flow against temperature gives an approximate straight line. This indicates a power-law relationship of the form $\beta T^{-\alpha}$ between temperature and flow. Different quartz tube orifices give different values of $\alpha$. The inset in Fig. 3 shows a log-log plot of the temperature dependence. A straight line is obtained with $\alpha=0.83$. However, when analyzing Eq. (4) an approximate power-law is found only for temperatures close to absolute zero with $\alpha=0.5$ and for very high temperatures with $\alpha=1$. For realistic temperatures, a straight line in a loglog plot can be found only for a sufficiently small temperature interval. The value of $\alpha$ is between 1 and 0.5 depending on the dimensions of the capillary, the pressures, and the temperature interval.

In conclusion, it is possible to produce micrometer-scale capillaries for introduction of gas into vacuum systems using deep reactive ion etching. In particular, it is possible to introduce gas to pressures compatible with mass spectrometers. Further, it is shown that for the parameters in question, the flow through the fabricated capillaries is described accurately by Knudsen intermediate flow. Based on this understanding, it is concluded that no transient flow or separation effects are present in the capillaries, so that the gas sampled with the capillary has the same composition as the gas to be analyzed.

The capillaries presented here might be particularly useful for gas analysis in combination with microfabricated chemical reactors. ${ }^{12}$ They can be directly integrated in the reactor design, and the very small amount of gas flowing to the mass spectrometer is compatible with the small quantities flowing in the microreactors. Further, the response time between changes in the reactor and measurements in the mass spectrometer will be minimized, enabling real-time measurements of reaction rates with rapidly changing reaction conditions. $^{13}$

\section{ACKNOWLEDGMENT}

We thank Martin Johansson for stimulating discussions and suggestions.

${ }^{1}$ B. Kasemo, Rev. Sci. Instrum. 50, 1602 (1979).

${ }^{2}$ S. Dahl, P. A. Taylor, E. Törnqvist, and I. Chorkendorff, J. Catal. 178, 679 (1998).

${ }^{3}$ C. Werdinius, L. Österlund, and B. Kasemo, Langmuir 19, 458 (2003).

${ }^{4}$ M. Johansson and L.-G. Ekedahl, Appl. Surf. Sci. 180, 27 (2001).

${ }^{5}$ S. Johansson, E. Fridell, and B. Kasemo, J. Vac. Sci. Technol. A 18, 1514 (2000).

${ }^{6}$ P. Norberg, L.-G. Petersson, and I. Lundström, Vacuum 45, 139 (1994).

${ }^{7}$ I. Lundström, P. Norberg, and L.-G. Petersson, J. Appl. Phys. 76, 142 (1994).

${ }^{8}$ P. Norberg, U. Ackelid, I. Lundström, and L.-G. Petersson, J. Appl. Phys. 81, 2094 (1997).

${ }^{9}$ A. M. Hynes, H. Ashraf, J. K. Bhardwaj, J. Hopkins, I. Johnston, and J. N. Shepherd, Sens. Actuators, A 74, 13 (1999).

${ }^{10}$ M. Johansson, I. Lundström, and L.-G. Ekedahl, Rev. Sci. Instrum. 71, 3513 (2000).

${ }^{11}$ A. Roth, Vacuum Technology, 3 ed., (Elsevier Science, New York, 1990).

${ }^{12}$ K. F. Jensen, Chem. Eng. Sci. 56, 293 (2001).

${ }^{13}$ J. Brandner, M. Fichtner, K. Schubert, M. Liauw, and G. Emig, Microreaction Technology: IMRET 5; Proc. of the 5th Internat. Conf., Strasbourg, (Springer, Berlin, 2001), pp. 167-174. 